\title{
Target direction rather than position determines oculomotor expectation in repeating sequences
}

\section{Authors:}

Andrew J. Anderson,* Matthew J. Stainer*, Peter Brotchie** \& R. H. S. Carpenter ${ }^{\dagger}$

* Department of Optometry and Vision Sciences, The University of Melbourne, 3010, Australia

** Clinical School, St. Vincent's Hospital, Fitzroy, 3065, Australia

${ }^{\dagger}$ University of Cambridge, Gonville and Caius College, Cambridge CB2 1TA, UK

Corresponding Author: Andrew J Anderson, Department of Optometry \& Vision

Sciences, The University of Melbourne, 3010, Australia. E-mail: aaj@unimelb.edu.au

Number of Figures, Tables \& Equations: 5 figures only

Total number of words:

i) Manuscript: 3,466

ii) Abstract: 197

Keywords: reaction time; saccade; sequence; disruption; inhibition 


\begin{abstract}
Saccadic latencies to targets appearing to the left and right of fixation in a repeating sequence are significantly increased when a target is presented out of sequence. Is this because the target is in the wrong position, the wrong direction, or both? To find out, we arranged for targets in a horizontal plane occasionally to appear with an unexpected eccentricity, though in the correct direction: this had no significant effect on latency, unlike what is observed when targets appeared in the unexpected direction. That subjects learnt sequences of directions rather than simply positions was further confirmed in an experiment where saccade direction was a repeating sequence but eccentricity was randomized: latency was elevated when a target was episodically presented in an unexpected direction. Latencies were also elevated when targets appeared in the correct hemifield but at an unexpected direction $\left(35^{\circ}\right.$ polar angular displacement from the horizontal, a displacement roughly equivalent in collicular spacing to our unexpected eccentricity), although this elevation was of a smaller magnitude than when targets appeared in an unexpected direction along the horizontal. Finally, we confirmed that not all changes in the stimulus cause disruption: an unexpected change in the orientation or colour of the target did not alter latency. Our results show that in a repeating sequence, the oculomotor system is primarily concerned with predicting the direction of an upcoming eye movement rather than its position. This is consistent with models of oculomotor control developed for randomly appearing targets in which the direction and amplitude of saccades are programmed separately.
\end{abstract}




\section{Introduction}

Many tasks in the natural world involve a set temporal sequence of behaviours (Hikosaka and Masaki 2008), for example when making a cup of tea (Land et al. 1999). It has been proposed that habitual sequences are controlled by parts of the motor system involved in controlling automated actions (Hikosaka et al. 1999), freeing cognitive processes for other, non-habitual tasks. Habitual sequences can, however, be episodically interrupted when an unexpected event breaks our routine - suddenly we need to suppress further automatic actions and engage conscious control mechanisms to allow us to get back on track. We have shown that a target appearing out of sequence (an interruption) in an oculomotor task produced elevated saccadic latencies (a disruption) both at the time of the interruption and for a period afterwards until the sequence is regained by the observer (Anderson and Carpenter 2010). Our paradigm used targets appearing at a fixed eccentricity either to the left or right of fixation, with the interruption appearing on the opposite side to that expected from the sequence. The precise cause of the disruption to saccadic latencies is ambiguous, however: is the disruption primarily the result of the altered position of the target or the altered direction of the saccade, or both? Moreover, could any unexpected event - such as a change in the target's physical characteristics - similarly result in a disruption?

The role of direction and position in saccadic programming has been extensively investigated, although primarily for randomly appearing targets. Holistic models of saccade programming propose that a neurological 'motor map' exists, where all potential movements are coded (Sparks 1988). A saccade is initiated when a certain location in the motor map is sufficiently activated (Findlay and Walker 1999), with saccades programmed as a single vector. In contrast, other researchers provide evidence that programming of the direction and amplitude of saccades are separable processes (Komoda et al. 1973; Becker and Jürgens 1979; Hou and Fender 1979; Aslin and Shea 1987; Abrams and Jonides 1988; Allik et al. 2003). For example, Komoda et al. (1973) used a pulse-step task where a target initially moved to one position but then rapidly to a different one. When a single saccade was made to the final position of a target, latencies were only increased if the saccade was in a different direction to that initially suggested by the initial target movement, with no cost found for changes in amplitude. The 
likelihood of needing to make a saccade to an unexpected location different from that indicated by the initial target movement is often high in studies investigating saccadic programming (Becker and Jürgens 1979), in contrast to the high predictability of target location and required saccadic response in sequence tasks (Anderson and Carpenter 2010).

Specific brain areas are thought to be involved in encoding sequences (Isoda and Tanji 2002; Lu et al. 2002) and suppressing a habitual sequence of responses that is no longer appropriate (Isoda and Hikosaka 2007). Whether models for saccadic programming determined with randomly appearing targets and randomly interleaved response types also apply to the habitual actions seen in oculomotor sequences is not known, as it has been suggested that how programming proceeds may depend upon the oculomotor task (Abrams and Jonides 1988). If saccadic direction is separately programmed in oculomotor sequences and participants are primarily learning the directions of the target in a sequence, then we would expect that an interruption to the expected direction of the target should influence latencies more than presenting a target in the correct direction but at a different location. There is preliminary evidence that the encoding of long-term expectation may be more concerned with the direction of the saccade for horizontally distributed targets, rather than its location, when targets are presented stochastically (Adams et al. 2000) consistent with work showing that certain aspects of visual attention divides hemispherically for horizontally located targets (Hughes and Zimba 1985; Tassinari et al. 1987). If, on the other hand, a spatial map provides the best account of saccade latencies in oculomotor sequences we would expect all types of interruptions to target position to increase latencies irrespective of saccade direction, as a new saccade would have to be programmed to the new location in the motor map.

In this paper we attempt to identify the relative importance of target position and direction in producing a disruption, and thereby investigate whether models of saccadic programming for stochastic targets may generalise to oculomotor sequences. Furthermore, we also investigate whether other changes to a target - such as altering its physical characteristics - can similarly cause a disruption. 


\section{Materials and Methods}

\section{Stimuli}

Targets in Experiments 1, 2 and 4 were $0.25^{\circ}$ diameter, $48 \mathrm{~cd} / \mathrm{m}^{2}$ dots viewed against a $24 \mathrm{~cd} / \mathrm{m}^{2}$ grey background. In Experiment 3, targets were triangles of $0.3^{\circ}$ width. For our supplementary experiment we used $14 \mathrm{~cd} / \mathrm{m}^{2}, 0.25^{\circ}$ diameter green $(\mathrm{CIE} 1931 \mathrm{x}=$ $0.624, y=0.341)$ or red $(x=0.288, y=0.608)$ spots. Targets were presented at either $4^{\circ}$ and $8^{\circ}$ in the horizontal plane from a fixation target placed at the centre of the screen for Experiments 1 through 3, as latencies are relatively constant for saccadic amplitudes between $0.75^{\circ}$ and $12^{\circ}$ (Kalesnykas and Hallett 1994).

\section{Apparatus}

Observers viewed stimuli on a calibrated computer system (ViSaGe graphics card: Cambridge Research Systems, Kent, UK, and Diamond Pro 2070SB monitor, Mitsubishi, Tokyo, Japan; frame rate $100 \mathrm{~Hz}$ ) subtending $23 \times 17$ degrees at the $1 \mathrm{~m}$ viewing distance. Horizontal eye position was recorded with an infrared reflection oculometer (Ober et al. 2003; Anderson et al. 2008), with saccades being detected automatically by SPIC software (Carpenter 1994) using a velocity threshold optimised for each observer. Head position was stabilised with a chin rest. Saccades were manually excluded if they contained irregularities such as blinks or head movements, and latencies less than $50 \mathrm{~ms}$ or greater than $790 \mathrm{~ms}$ were removed. The average percentages of removed trials were 12.5, 2.6, 7.1 and 4.9\% for Experiments 1 through 4, respectively.

\section{Experimental Protocols}

All experiments used a step task (Carpenter and Williams 1995) with the fixation target reappearing at the centre of the screen after a saccade had been detected. The next target appeared after a randomly selected delay uniformly distributed between $770 \mathrm{~ms}$ and $1770 \mathrm{~ms}$. Participants were explicitly informed of the structure of each experimental run in advance, including the probabilities involved, and were required to describe the structure 
accurately to the experimenter prior to data collection. Participants also completed a run from each experimental protocol for training purposes prior to data collection for each experiment.

Experiment 1: Targets appeared in a repeating pattern of four elements (either LLLR [Fig. 1, upper set] or RRRL) at a fixed eccentricity. On any trial during the sequence there was a $1 / 31$ probability of a target appearing out of sequence (an interruption) that could be one of two types. For interruptions of position and direction, the target appeared at the correct eccentricity but on the opposite side to that expected from the pattern. For interruptions of position alone, the target appeared on the correct side but not at the expected eccentricity. After each interruption the target could appear with equal probability to either the left or right of fixation at the same eccentricity used in the pattern for a single trial, meaning that subjects had to temporarily perform a task different from the learnt sequence immediately after a disruption, similar to previous investigations (Anderson and Carpenter 2010). The pattern then re-commenced from the beginning of the four-element sequence. Twelve runs of 110 trials were counterbalanced for eccentricity (4 and 8) and for the high-frequency side in the sequence (left or right).

Experiment 2: Participants viewed targets whose direction was a repeating pattern of four elements (either LLLR [Fig. 1, middle set] or RRRL) but whose eccentricities varied randomly between $4^{\circ}$ or $8^{\circ}$ with equal probability. On any trial during the sequence there was a 1/31 probability of an interruption occurring, where the target appeared on the incorrect side and with a random eccentricity $\left(4^{\circ}\right.$ or $\left.8^{\circ}\right)$. If participants primarily learn a sequence of saccadic directions, interruptions to direction in this experiment should still elevate latencies despite random changes in target position. After each interruption, a target appeared at random $4^{\circ}$ to either the left or right of fixation with equal probability, after which the pattern re-commenced from the beginning of the four-element sequence. Eight 
runs of 110 trials were counterbalanced for eccentricity for the high-frequency side in the sequence (left or right).

Experiment 3: This experiment was used to check if only specific types of changes to the target (e.g. direction) could cause disruptions, rather than any change. Targets appeared in a repeating pattern of four elements (either LLLR [Fig. 1, upper set] or RRRL) with the target being a triangle pointing downwards. Interruptions (with a probability of 1/31) occurred when the target appeared in the expected position, but was pointing upwards. Interruption trials were followed by a trial where the target (downward pointing triangle) appeared with equal probability either to the left or right of fixation, after which the pattern re-commenced from the beginning of the four-element sequence. Eight runs of 120 trials (all at $4^{\circ}$ eccentricity) were counterbalanced for the high-frequency side in the sequence (left or right). To check that any findings were not a special case restricted to orientation changes, in a supplementary experiment (four observers) we repeated Experiment 3 except with the expected target being a particular colour and the interruption being of the opposite colour. The expected colour was fixed for a given run, with red and green targets counterbalanced across the experiment.

Experiment 4: We used this experiment to check if changes in target direction within the expected hemifield anticipated from the sequence could also cause disruptions. The experiment proceeded similarly to Experiment 1, except that targets causing a direction interruption appeared in the anticipated hemifield but $35^{\circ}$ (in polar coordinates, relative to the central fixation point) randomly above the nominal target position. This direction interruption approximately matches our $4^{\circ}$ amplitude interruption (i.e. target appearing horizontally at $8^{\circ}$ rather than $4^{\circ}$ ), in terms of displacement on motor maps of the monkey colliculus (Ottes et al. 1986). Twelve runs of 110 trials were counterbalanced for the highfrequency side in the sequence (left or right), using a single eccentricity of $4^{\circ}$ for the uninterrupted sequence. As our oculometer could only measure the horizontal component 
of the oblique saccades used in this experiment and so could not check whether an appropriate vertical component had also been performed, participants were specifically instructed to try to accurately foveate each target.

In all our experiments, an interruption could occur on any trial except those containing random targets and those immediately following random targets. We deleted from our analysis any interruptions that occurred within five trials of each other, to ensure that a complete run of the uninterrupted sequence had occurred. We ignored the first 10 trials in each run.

Analysis

Data were analysed using linear mixed effect (LME) modeling techniques. These techniques have garnered increasing use, particularly within psycholinguistics (e.g. Kliegl (2007); Baayen et al. (2008)). The data were analysed using the lme4 (Bates \& Maechler: R package version 0.999375-32) and languageR (Baayen: R package version 0.955) packages in R studio. We examine the effect of different types of interruption on saccade latencies, with participant and the target's absolute position being random factors into the model. Although systematic changes in latency were not anticipated over the saccade amplitudes we investigated (Kalesnykas and Hallett 1994), we justified our inclusion of target position as a factor to account for idiosyncratic differences seen between individual participants (Fig. 1) that are of a magnitude that could mask the latency differences in sequence interruption experiments (Anderson and Carpenter 2010). Our data are therefore effectively normalized by the variation in latency seen in Figure 1 to stochastically presented targets, although it should be noted that the magnitude of any latency change due to an interruption of target position is not altered by this process. Figure 1 also highlights the need to include participant as a factor, as absolute saccadic latency varies between observers. We report the mean difference between conditions with highest $95 \%$ posterior 
density intervals from Markov Chain Monte Carlo mean estimates, with approximated $p$ values (Baayen 2008; Baayen et al. 2008).

In Experiment 4, targets that were rotated by $35^{\circ}$ appeared as interruptions only. Because of this, we cannot compare latencies to such targets presented as part of a sequence and as an interruption, unlike in our previous experiments. Therefore, for each individual we normalised latencies for each position to the harmonic mean latency for that individual at the position measured over four runs (440 trials total) in which targets were stochastically interleaved with equal probability. We then ran a linear mixed effect model on these normalised latencies, with participant entered as a random factor.

For Experiment 1, we also used a repeated measures ANOVA to determine whether saccadic amplitudes differed for saccades made during interruption trials versus those made to a target in the same position during the uninterrupted sequence.

\section{Participants}

We tested a common group of eight participants for Experiments 1 through 3, and a differently constituted group of eight participants for Experiment 4. All participants in Experiments 1 through 3 were required to have fewer than 5\% direction errors during a preliminary training period in order to be included, and had normal visual fields as assessed using the Humphrey Matrix perimeter (Anderson et al. 2005). None showed fixation errors when performing perimetry. In Experiment 4, appropriate fixation was assessed via an analysis of saccadic amplitudes (see Results, below). Authors AJA and MJS were participants in each experiment. All procedures were in accordance with the 1964 Declaration of Helsinki and were approved by the University of Melbourne ethics committee. Participants gave informed consent prior to inclusion in the study. 


\section{Results}

The results from Experiment 1 are shown in Fig. 3. An interruption to both target position and direction (upper panel) caused a significant elevation of latency $(p<0.001)$, with latencies not different from baseline upon the return of the sequence. In contrast, an interruption to target position alone (lower panel) did not significantly elevate latencies ( $p=0.13$ ). The $95 \%$ confidence intervals surrounding the latencies for position and direction interruptions did not overlap, indicating that disruption was significantly greater for direction interruptions. The amplitude of saccades were not different for interruption trials versus saccades to the same target location made during uninterrupted portions of the sequence $(F<1)$.

Figure 4 shows the results from Experiment 2. Despite target eccentricity being randomized each trial, an interruption to the expected response direction still significantly elevated latencies $(p<0.001)$. The magnitude of the disruption was significantly less $(p<0.001)$ than for the target direction interruptions investigated in Experiment 1 (Fig. 3).

The results of Experiment 3 are shown in Figure 5. An interruption to target orientation alone failed to significantly change latencies $(p=0.8)$. Our supplementary experiment showed that interruptions based on target colour gave similar results, with no significant change in latency at the time of the interruption (mean elevation $=1.9 \mathrm{~ms}, p=0.44$ ).

Figure 6 shows the results of Experiment 4. Although an interruption of saccadic amplitude produced no significant disruption to latency (lower panel), consistent with Experiment 1, an interruption to the polar angular direction of the target significantly disrupted latencies $(p<0.001)$. The $95 \%$ confidence intervals around the direction interruption magnitudes seen in Experiment 1 (28.3 ms; 95\% CI 22.6 to $33.9 \mathrm{~ms})$ and Experiment 4 (16.0 msms; 95\% CI 12.1 to $20.1 \mathrm{~ms}$ ) did not overlap, indicating that the disruption was significantly less for targets rotated away from the horizontal than for targets appearing in the unexpected 
hemifield. The horizontal amplitudes of saccades to our rotated targets were significantly smaller (two-tailed $t$-test, $p<0.05$ for each participant) than amplitudes to targets in the uninterrupted portion of the sequence, as would be anticipated if participants reliably fixated the targets.

\section{Discussion}

We have previously demonstrated that a target appearing out of sequence can elevate saccadic latencies (Anderson and Carpenter 2010). Here we identify the aspects of the stimulus that are critical for causing this disruption. When a target was presented in an unexpected direction (and, necessarily, position), latencies were significantly longer (Fig. 3, upper panel). When the target was presented in an unexpected location, but one where the direction of the expected saccade was unaltered, latencies were not significantly altered (Fig. 3, lower panel). Directly compared, latency elevation was greater for an interruption to direction rather than position alone (Fig 3., non-overlapping confidence intervals at interruption). Even when direction interruptions involved targets appearing in the correct hemifield and at a position designed to match the size of our amplitude interruptions in collicular coordinates, a significant interruption occurred (Fig. 6) albeit of a smaller magnitude than when targets appeared in the unexpected hemifield. Taken together, these results show it is a change in the direction of where a target appears that is critical in causing a disruption to saccadic latency, particularly when the change of direction is large (e.g. to the opposite hemifield). This would suggest that learning a sequence of response directions is of critical importance in encoding sequences of oculomotor reponses. Further support for this position comes from our Experiment 2, in which the target sequence was encoded purely by direction, with target position (eccentricity) randomized on each trial. Such a sequence still demonstrated the characteristic latency disruption when an interruption occurred (Fig. 4). Finally, we confirmed that any type of interruption will not 
necessarily cause a disruption, as changing the orientation (Fig. 5) or colour of the target had no effect on saccadic latencies.

Although our Experiment 1 failed to show a significant disruption to saccadic latency when horizontal target position alone was altered, position may still have some role in encoding sequences. When we randomised position in Experiment 2, the latency elevation seen when a disruption to the expected response direction occurred was significantly less than in Experiment 1. This suggests that a sequence of response directions alone (Expt. 2) is less compelling than a sequence of response directions linked to specific positions (Expt. 1), and so interruptions to such a sequence do not produce as marked latency elevations. Previous work has suggested that target location is important for learning sequences in manual tasks, although no clear consensus yet exists on the relationship between stimulus and response in learning sequences (Abrahamse et al. 2010). It has been argued (Hikosaka et al. 1999) that parallel networks exist for learning sequential procedures, with initial learning being more dependent on spatial learning, and control then taken by relatively automatic parts of the motor system as sequences become habitual. Indeed, the brain areas most activated in humans during sequence learning appear different from when a sequence is mastered (Hikosaka et al. 1996; Sakai et al. 1998). The sequences investigated here were simple and of short duration, and so are presumably rapidly learnt and automated. Furthermore, our protocol took specific steps to facilitate this learning (i.e. having subjects explicitly describe the structure of the protocol prior to testing, performing a complete training run for each protocol, and discarding the first section of each experimental run analysed). The structure of our Experiment 2 necessarily meant that encoding saccadic amplitude could not be automated however, and so a role for stimulus-based effects of target position would remain in this experiment even when learning was complete. 


\section{Programming of saccadic eye movements}

There have been various mechanisms in which the two principal parameters of a saccade amplitude and direction - might be programmed for randomly appearing targets. It is instructive to consider if these programming mechanisms can be applied to our data from oculomotor sequences. Parameters might be programmed serially (e.g. Becker and Jürgens (1979)) or in parallel (e.g. Abrams and Jonides (1988)). If our findings are caused by a parallel mechanism, we require the assumption that reprogramming direction takes longer than reprogramming amplitude in order to explain why an unexpected direction disrupted latency more than an unexpected amplitude. Such an account would be consistent with findings of Allik, Toom and Luuk (2003), who found that when saccade parameters were programmed in isolation (with advance knowledge of the other parameter), amplitude programming was faster than direction programming. If our findings are the result of a serial mechanism we would need to assume that direction is programmed in advance of amplitude, as seen in models such as that by Becker and Jürgens (1979). When an unexpected amplitude only is encountered, only the second half of the saccade would need to be reprogrammed, thereby minimizing the disruption to latency. If amplitude were encoded first however, an unexpected amplitude would require both amplitude and direction to be reprogrammed and so disruption to latencies would be maximized in this condition, contrary to our findings. That direction programming precedes amplitude programming has been found by some (Becker and Jürgens 1979; Aslin and Shea 1987), but not all (Abrams and Jonides 1988), authors. Despite much early work examining the ordering and timing of those steps programming saccade amplitude and direction, subsequent work (Allik et al. 2003) seems to suggest a much more dynamic relationship between saccadic amplitude and direction programming than originally posited, adding further to the already acknowledged limitations of simplified saccadic programming models (Becker and Jürgens 1979). Furthermore, programming may depend upon the oculomotor task used, with Abrams and Jonides (1988) showing amplitude and direction 
being separately programmed for non-reflexive tasks (i.e. made to an existing target in response to a reaction cue) but being more holistically programmed for more reflexive saccades.

That latency disruption appears primarily based on the direction of the required eye movement in a sequence is consistent with the idea that oculomotor direction is separately programmed, and is inconsistent with what we would predict based on a simple motor map (see Introduction). Our findings do not discount the idea of an oculomotor map at some stage within the oculomotor pathway however - for example, the superior colliculus (Carpenter 2000) - but do suggest that those mechanisms that produce disruptions in oculomotor sequences may not act at this stage. The results of our Experiment 4 (Fig. 6) certainly make the superior colliculus a very unlikely candidate, unless the spatial pattern of activation associated with programming a saccade shows marked anisotropies within the collicular map - in contrast to the Gaussian pattern of activation assumed in modelling (van Opstal and van Gisbergen 1989) - or that significant differences exist between collicular maps for monkeys and humans. Motor maps and those mechanisms responsible for programming amplitude and direction may exist as two serial stages, wherein the final destination of the eye (as represented on a motor map) is then translated in to the appropriate programming commands for the eye movement to occur (Allik et al. 2003). Analysis of human data suggests that saccadic amplitudes are subject to greater uncertainty than direction, and that part of this uncertainly may arise in post-colliculular processing stages (van Opstal and van Gisbergen 1989).

Previous work has suggested that oculomotor expectation appears to be encoded grossly into spatial hemifields (Adams et al. 2000) and some findings suggest that saccadic reprogramming only increases latency when the horizontal direction of the programmed saccade changes (Komoda et al. 1973). Both of these former observations were made with targets whose appearance was stochastic. Specific areas of the brain have been implicated 
in encoding sequences (Isoda and Tanji 2002; Lu et al. 2002) and suppressing inappropriate habituated responses that are no longer appropriate (Isoda and Hikosaka 2007), such as when an interruption to a sequence occurs. Despite these specific brain processes, our current findings suggest the direction-specific nature of expectation seen when making saccades to stochastically presented targets (Adams et al. 2000) also applies to when targets appear within a learnt patterned sequence. This is consistent with models for saccadic generation where encoding of saccadic amplitude and direction occurs comparatively late, and so is performed in a common way irrespective of the initiating stimulus (e.g. visual, memory, or automated) (Findlay and Walker 1999). Imaging studies also provide evidence for common processing pathways for certain types of saccadic eye movements (Petit et al. 1996). Our results suggest that disruptions are not restricted to changes in the hemifield for learnt sequences, as even polar angular changes in direction where the target appears in the anticipated hemifield still produce a disruption. It is possible that expectation encoding (Adams et al. 2000) and saccadic programming (Komoda et al. 1973) for stochastic targets may similarly show effects not grossly limited to spatial hemifield if targets other than horizontally displaced ones were employed. Our results do, however, suggest that disruption is increased when targets appear in the unexpected hemifield. Given that the left superior colliculus encodes saccades to the right, and vice versa, this additional disruption may be unrelated to any cost of altering programming location within an oculomotor map but rather reflect a cost of switching to an alternative map belonging to the other hemisphere.

In conclusion, our results demonstrate that interruptions to learnt oculomotor sequences result principally from changes to the expected direction of the target requiring a response, rather than its position. This finding is consistent with a view that direction of action rather than amplitude - is of principal importance when programming each saccade in a habitual sequence, in accordance with some previous models of the programming of saccades to stochastically appearing targets. 


\section{Acknowledgements}

This work was supported by Australian Research Council Project Grant DP120100651. 

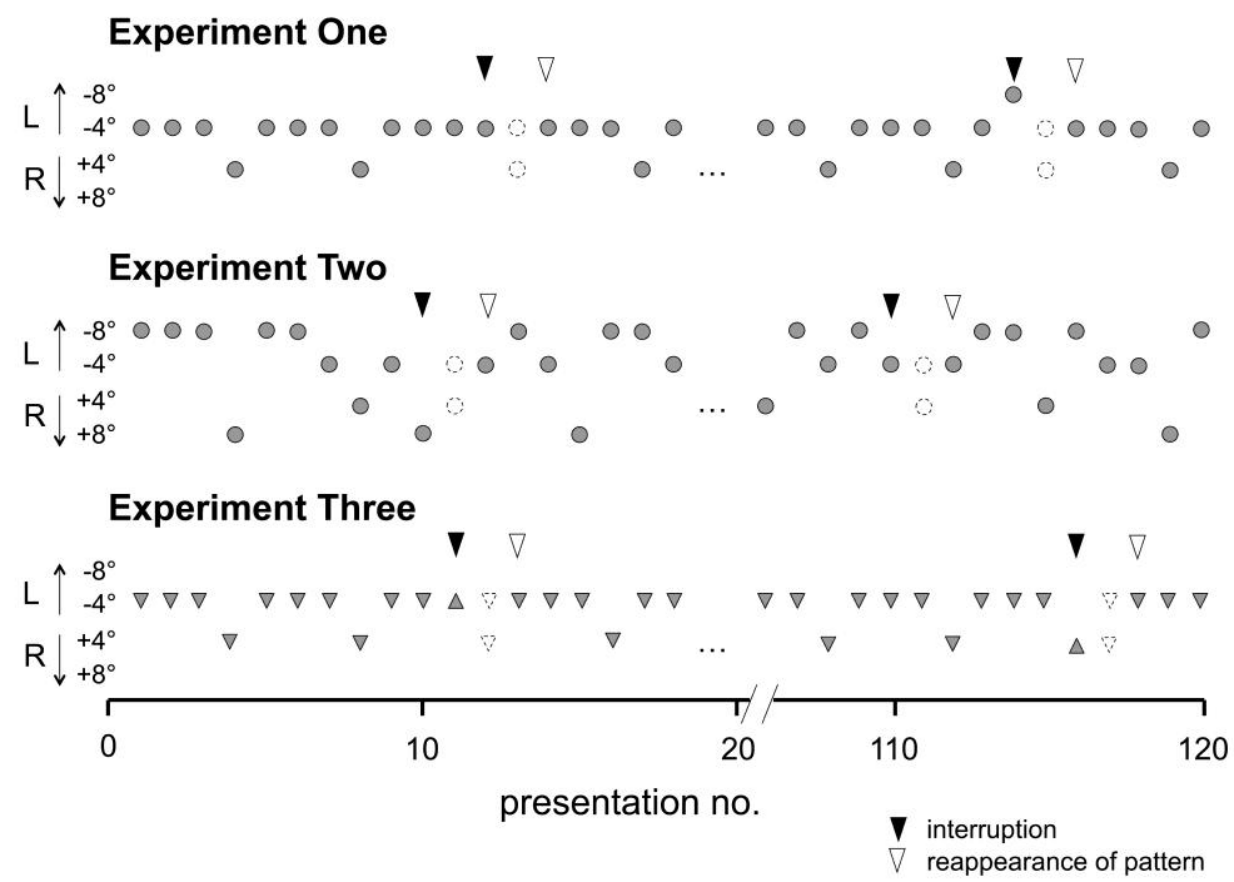

Figure 1. Examples of the target sequences used in each experiment. After each interruption to the sequence (black triangles) targets could appear in one of two locations (dashed symbols) with equal probability. In Experiment One, interruptions were either to target position and direction (first interruption on figure) or to target position alone (second interruption on figure). In Experiment Two, the repeating pattern and interruptions were based solely on target direction, with eccentricity being randomized. In Experiment Three, interruption targets appeared at the correct position but with a different form (upward rather than downward pointing triangles). All targets were presented as part of a step task, wherein a central fixation marker disappeared when the peripheral target appeared: after a saccade was detected the target disappeared, the fixation marker reappeared, and the subject returned their gaze to the fixation marker. In the actual experiments, the lowfrequency side was to the left as often as it was to the right, and the eccentricity of the pattern in Experiment One was counterbalanced between runs of $4^{\circ}$ and of $8^{\circ}$. 


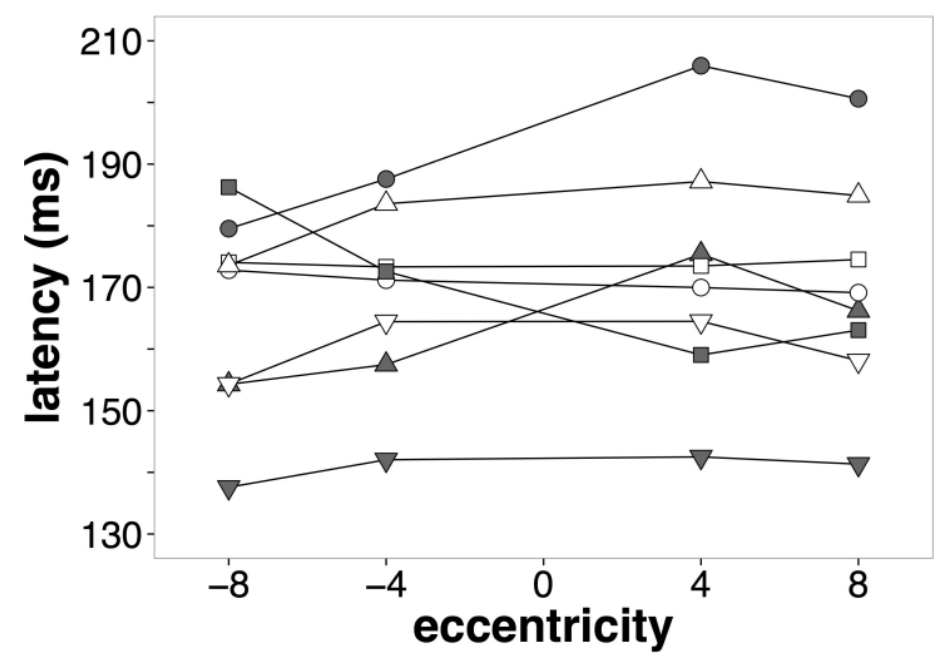

Figure 2. Mean latencies for each of the eight observers, for targets appearing stochastically and with even probability to each of four positions. Data was collected in 2 runs of 120 trials, with the first 20 from each run excluded. Data points represent harmonic means. Here and in the following figures, unfilled squares and unfilled circles show data from authors (AJA \& MJS, respectively). 


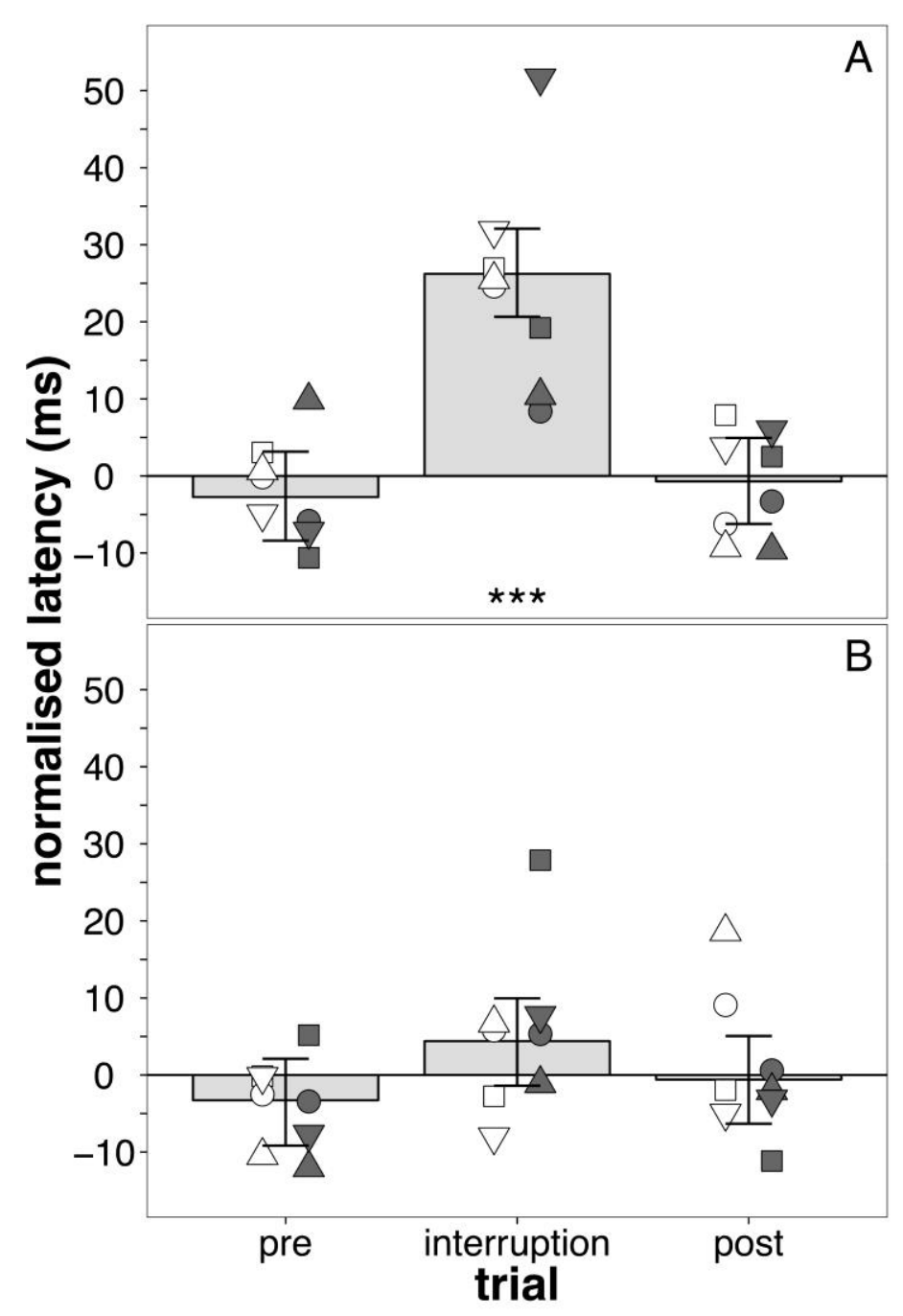

Figure 3. Results from Experiment 1, where episodic interruptions were made to either the expected direction and position of the target (Panel A), or to the target position alone (Panel B). The grey bars show changes in latency, averaged across all subjects, immediately before an interruption ("pre"), at the interruption ("interruption"), and immediately upon returning to the sequence ("post"). Changes are normalised to latencies obtained during the uninterrupted sequence (average latency $=171.5 \mathrm{~ms}$ ), with error bars giving 95\% confidence intervals. Asterisks indicate latencies that are significantly different from the uninterrupted portion of the sequence $(* p<0.05, * * p<0.01$, *** $p<0.001)$. Mean direction errors (i.e. saccades made in the direction opposite to the target) were $2.9 \%$. Individual participant data is represented with symbols, with symbol type being consistent across Figures $4 \& 5$. For each participant, latency distributions during the sequence 
(excluding interruption trials, and the five trials immediately following) were unimodal (see Supplementary Material). 


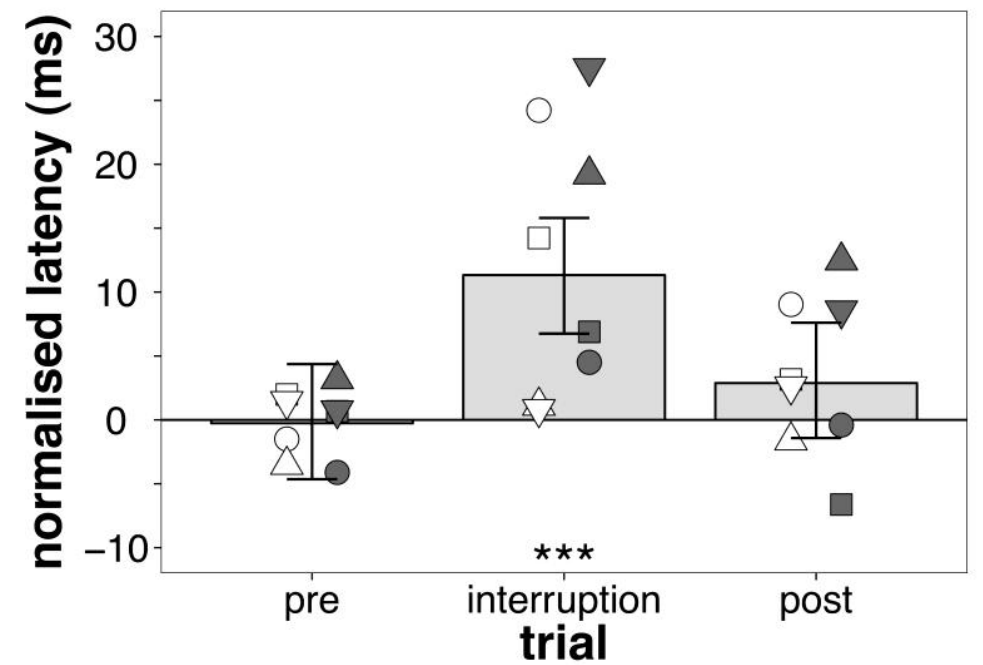

Figure 4. Results from Experiment 2, where episodic interruptions were made to the expected response direction whilst target eccentricity was randomised on each trial. The mean latency used for normalisation was $180.5 \mathrm{~ms}$, and mean direction errors were $0.6 \%$. Other details are as given in Fig. 3. 


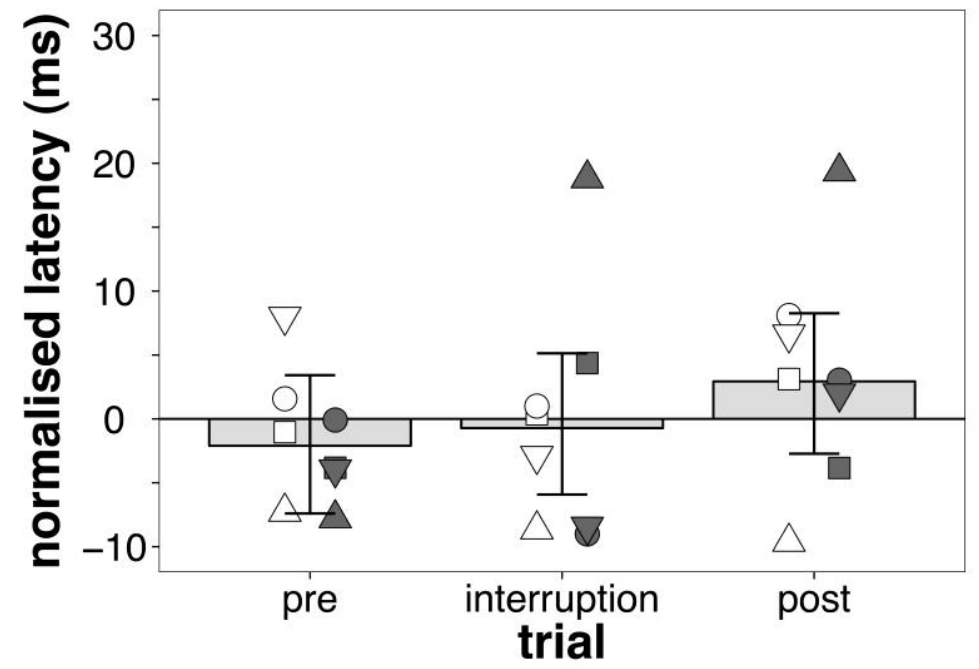

Figure 5. Results from Experiment 3, where episodic interruptions were made to the expected orientation of a triangle target, with the response direction and target position unchanged. The mean latency used for normalisation was $180.5 \mathrm{~ms}$, and mean direction errors were 3.3\%. Other details are as given in Fig. 3. 


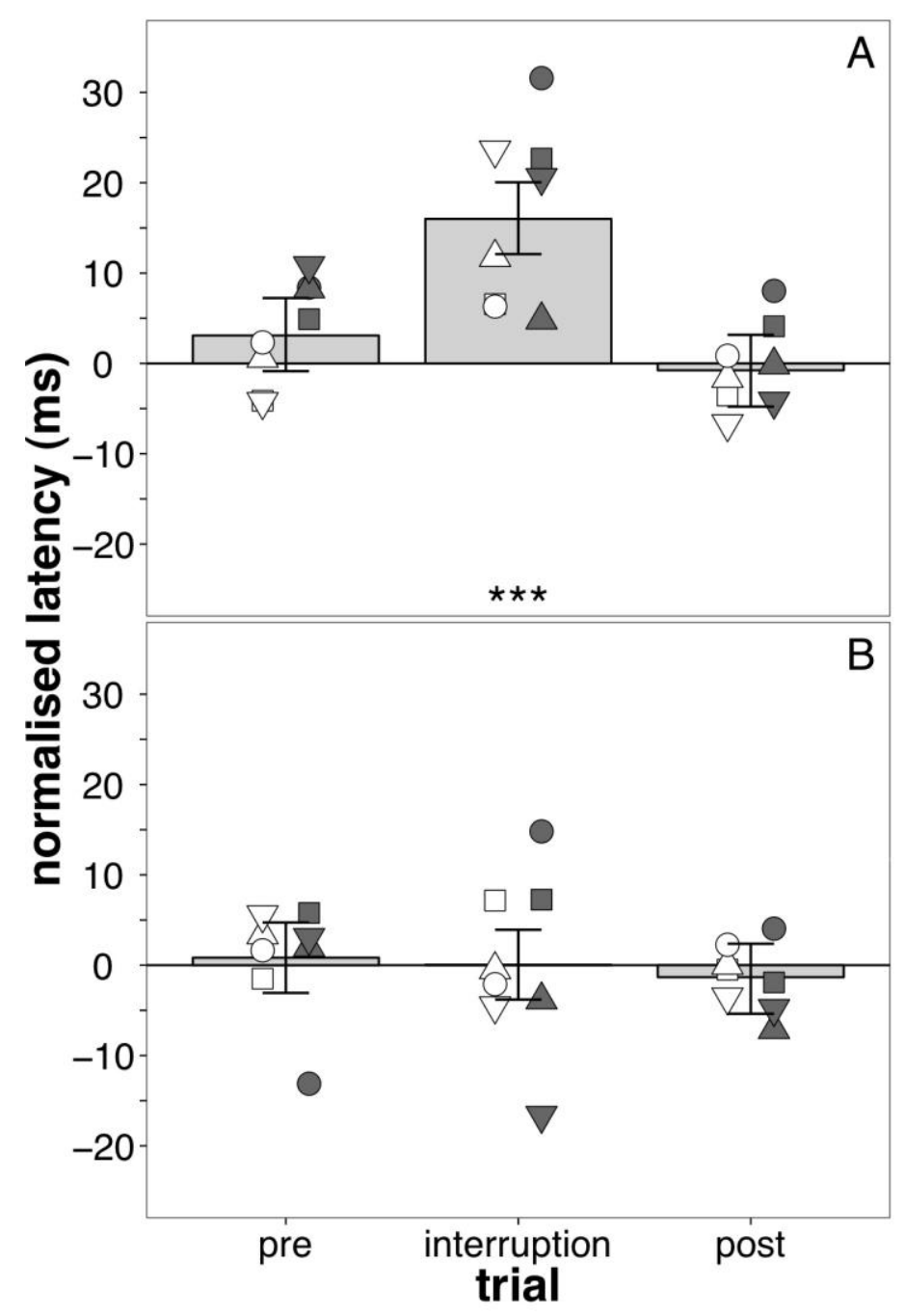

Figure 6: Results from Experiment 4, where episodic interruptions were made to either the expected direction of the target (Panel A: saccade amplitude of $4^{\circ}$, but at a polar angle of $35^{\circ}$ above the horizontal axis), or to the amplitude of the required saccade (Panel B: target at $8^{\circ}$ along the horizontal axis). Participants made no direction errors. Individual participant data is represented with symbols, although symbols do not correspond with those used in Figs 3 through 5. Other details are as given in Fig 3. 


\section{References}

Abrahamse EL, Jiménez L, Verwey WB, Clegg BA (2010) Representing serial action and perception. Psychonomic Bulletin \& Review 17:603-623

Abrams RA, Jonides J (1988) Programming saccadic eye movements. Journal of Experimental Psychology: Human Perception and Performance 14:428-443

Adams MWJ, Wood D, Carpenter RHS (2000) Expectation acuity: the spatial specificity of the effect of prior probability on saccadic latency. Journal of Physiology 527(P):140-141

Allik J, Toom M, Luuk A (2003) Planning of saccadic eye movements. Psychological Research 67:10-21 doi: 10.1007/s00426-002-0094-5

Anderson AJ, Carpenter RHS (2010) Saccadic latency in determinisitic environments: getting back on track after the unexpected happens. Journal of Vision 10:1-10

Anderson AJ, Johnson CA, Fingeret M, Keltner JL, Spry PGD, Wall M, Werner JS (2005) Characteristics of the normative database for the Humphrey Matrix perimeter. Investigative Ophthalmology and Visual Science 46:1540-1548

Anderson AJ, Yadav H, Carpenter RHS (2008) Directional prediction by the saccadic system. Current Biology 18:614-618

Aslin RN, Shea SL (1987) The amplitude and angle of saccades to double-step target displacements. Vision Research 27:1925-1942

Baayen RH (2008) Analyzing linguistic data: A practical introduction to statistics. Cambridge University Press, Cambridge

Baayen RH, Davidson DJ, Bates DM (2008) Mixed-effects modeling with crossed random effects for subject and item. Journal of Memory and Language 59:390-412

Becker W, Jürgens R (1979) An analysis of the saccadic system by means of double step stimuli. Vision Research 19:967-983

Carpenter RHS (1994) SPIC: a PC-based system for rapid measurements of saccadic responses. Journal of Physiology (Proceedings) 480

Carpenter RHS (2000) The neural control of looking. Current Biology 10:R291-R293 
Carpenter RHS, Williams MLL (1995) Neural computation of log likelihood in control of eye movements. Nature 377:59-62

Findlay JM, Walker R (1999) A model of saccade generation based on parallel processing and competitive inhibition. Behavioral and Brain Sciences 22:661-721

Hikosaka O, Masaki I (2008) Brain mechanisms for switching from automatic to controlled eye movements. Progress in Brain Research 171:375-382

Hikosaka O, Nakahara H, Rand MK, et al. (1999) Parallel neural networks for learning sequential procedures. Trends in Neurosciences 22:464-471

Hikosaka O, Sakai K, Miyauchi S, Takino R, Sasaki Y, Pütz B (1996) Activation of human presupplementary motor area in learning of sequential procedures: a functional MRI study. Journal of Neurophysiology 76:617-621

Hou RL, Fender DH (1979) Processing of direction and magnitude by the saccadic eyemovement system. Vision Research 19:1421-1426

Hughes HC, Zimba LD (1985) Spatial maps of directed visual attention. Journal of Experimental Psychology: Human Perception and Performance 11:409-430

Isoda M, Hikosaka O (2007) Switching from automatic to controlled action by monkey medial frontal cortex. Nature Neuroscience 10:240-248

Isoda M, Tanji J (2002) Cellular activity in the supplementary eye field during sequential performance of multiple saccades. Journal of Neurophysiology 88:3541-3545

Kalesnykas RP, Hallett PE (1994) Retinal eccentricity and the latency of eye saccades. Vision Research 34:517-531

Kliegl R (2007) Toward a perceptual-span theory of distributed processing in reading: a reply to Rayner, Pollatsek, Drieghe, Slattery, and Reichle (2007). Journal of Experimental Psychology: General 136:530-537

Komoda MK, Festinger L, Phillips LJ, Duckman RH, Young RA (1973) Some observations concerning saccadic eye movements. Vision Research 13:1009-1020

Land MF, Mennie NR, Rusted J (1999) The roles of vision and eye movements in the control of activities of daily living. Perception 28:1311-1328 
Lu X, Matsuzawa M, Hikosaka O (2002) A neural correlate of oculomotor sequences in supplementary eye field. Neuron 34:317-325

Ober JK, Przedpelska-Ober E, Gryncewicz W, Dylak J, Carpenter RHS, Ober JJ (2003) Hand-held system for ambulatory measurement of saccadic durations of neurological patients. In: Gajda J (ed) Modelling and Measurement in Medicine. Komitet Biocybernityki i Inzyneierii Biomedycznej PAN, Warsaw, pp 187-198

Ottes FP, van Gisbergen JA, Eggermont JJ (1986) Visuomotor fields of the superior colliculus: a quantitative model. Vision Research 26

Petit L, Orssaud C, Tzourio N, Crivello F, Berthoz A, Mazoyer B (1996) Functional anatomy of a prelearned sequence of horizontal saccades in humans. The Journal of Neuroscience 16:3714-3726

Sakai K, Hikosaka O, Miyauchi O, Takino R, Sasaki Y, Pütz B (1998) Transition of brain activation from frontal to parietal areas in visuomotor sequence learning. The Journal of Neuroscience 18:1827-1840

Sparks DL (1988) Neural cartography: sensory and motor maps in the superior colliculus. Brain, Behavior and Evolution 31:49-56

Tassinari G, Aglioti S, Chelazzi L, Marzi CA, Berlucchi G (1987) Distribution in the visual field of the costs of voluntarily allocated attention and of the inhibitory aftereffects of covert orienting. Neuropsychologia 25:55-71

van Opstal AJ, van Gisbergen JAM (1989) Scatter in the metrics of saccades and properties of the collicular motor map. Vision Research 29:1183-1196 